\title{
Optical-radar imaging of scale models for studies in asteroid astronomy
}

\author{
A. K. Andrews and R. S. Hudson \\ School of Electrical Engineering and Computer Science, Washington State University, Pullman, Washington $99164-2752$ \\ D. Psaltis \\ Department of Electrical Engineering, California Institute of Technology, Pasadena, California 99125
}

Received July 17, 1995

\begin{abstract}
During the past five years, delay-Doppler radar has become the primary technique for studying the structure of Earth-crossing asteroids. None of these objects has yet been visited by spacecraft, so ground-truth test cases are lacking. A laboratory system is described that provides optical-radar images at 0.1-mm resolution. These data are analogous to the highest-resolution asteroid radar images currently available and provided realistic test cases for developing signal-processing techniques. The system can be thought of as a 1/188,000 scale model of the Arecibo radar, or a 1/52,800 scale model of the Goldstone radar. ( 1995 Optical Society of America
\end{abstract}

Earth-crossing asteroids (ECA's) are of increasing interest because of the collision hazard that they pose to Earth $^{1}$ and as the logical next steps for human exploration beyond the Earth-Moon system. ${ }^{2}$ Planning for both hazard mitigation and exploration can be significantly aided by detailed physical models of these objects. Ground-based delay-Doppler (DD) radar (also called range-Doppler radar) has become the primary technique for obtaining structural information for these small bodies, and improvements in planetary radar systems and an increasing discovery rate for ECA's guarantee that the number and quality of ECA DD radar observations will continue to grow in coming years. Yet the relation between an object's shape and its DD image(s) is nonintuitive, and the development of interpretive and signal-processing techniques for these data has been hampered by the lack of ground truth test cases. $^{3}$ Spacecraft missions to a representative sample of radar-detected asteroids would be the ideal way to gather such data, but this is not currently an option. A more practical approach is to build a scale model of a radar-asteroid system in the laboratory. In this Letter we report on the application of an optical-radar system to asteroid radar studies.

A radar telescope resolves in time delay $\tau$ and Doppler frequency $\nu$. These correspond to distances along, respectively, the line of sight and the planeof-sky (POS) direction, which is orthogonal to the object's instantaneous apparent spin vector. Figure 1 diagrams the relevant geometry, where the $z$ axis points out of the page toward the radar and the $y$ axis is the projection of the spin vector on the POS. Viewed on the POS, i.e., the $x-y$ plane, isoDoppler contours, the locus of all points with a given radial velocity relative to the radar, are lines of constant $x$; iso-delay contours, the locus of all points at a given distance from the radar, are lines of constant $z$ and depend on the object's shape. More precisely,

$$
\begin{aligned}
\tau(x, y) & =-\frac{2}{c} z(x, y), \\
\nu(x) & =-\frac{4 \pi \cos \delta}{\lambda_{r} P} x,
\end{aligned}
$$

where $c$ is the speed of light, $\lambda_{r}$ is the radar wavelength, $P$ is the rotation period, and $\delta$ is the subradar latitude. ${ }^{4}$

Each pixel of a DD image results from the sum of the POS brightness at all points of intersection of the corresponding delay and Doppler contours (e.g., filled circles in Fig. 2). The resulting DD image can be described by the equation

$$
\sigma(\tau, \nu)=\left|\int h_{\tau}[\tau-\tau(x, y)] h_{\nu}[\nu-\nu(x)] b(x, y) \mathrm{d} x \mathrm{~d} y\right|^{2}
$$

where $b(x, y)$ is the complex POS brightness of the object and the system impulse responses are typically

$$
\begin{aligned}
& h_{\tau} \propto\left\{\begin{array}{cl}
1-|\tau / \Delta \tau| & \tau \leq \Delta \tau, \\
0 & \tau>\Delta \tau
\end{array}\right. \\
& h_{\nu} \propto \operatorname{sinc}(\nu / \Delta \nu)
\end{aligned}
$$

for delay and Doppler resolutions $\Delta \tau$ and $\Delta \nu$. The random-phasor-sum nature of the integrand results in speckle, or self-noise. In the limit that a very large number of these single looks (each with a slightly different value of $\psi$ ) are averaged, we arrive at

$$
\begin{aligned}
\langle\sigma(\tau, \nu)\rangle= & \int\left|h_{\tau}[\tau-\tau(x, y)] h_{\nu}[\nu-\nu(x)]\right|^{2} \\
& \times\left\langle|b(x, y)|^{2}\right\rangle \mathrm{d} x \mathrm{~d} y .
\end{aligned}
$$




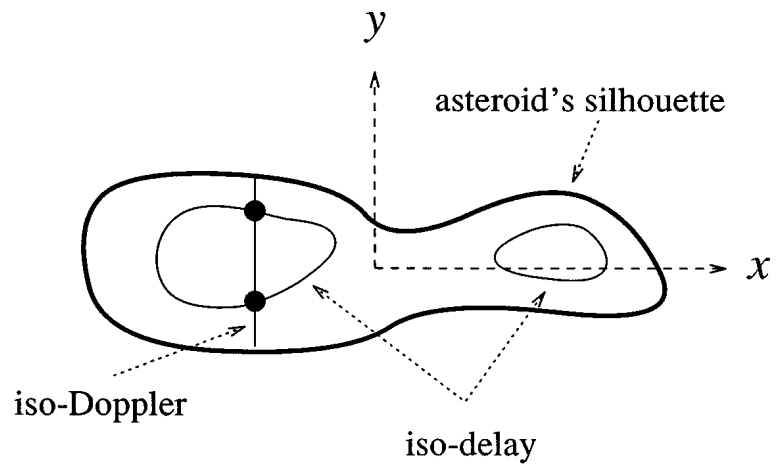

Fig. 1. DD imaging geometry.

Clearly, any system that can provide $b(x, y)$, $\left\langle|b(x, y)|^{2}\right\rangle$, and $z(x, y)$ can provide $\sigma(\tau, \nu)$ and $\langle\sigma(\tau, \nu)\rangle$.

The close analogy between coherence length measurement in a Michelson interferometer and pulse compression in a radar system has been exploited by many authors to achieve range resolution with optical sources. Dresel et al. pointed out that the introduction of an imaging system effectively results in many interferometers operating in parallel, thereby permitting range resolution for each pixel in an image, i.e., measurement of $z(x, y){ }^{5} \quad$ Hudson suggested that a three-dimensional imaging system of this sort could be used to simulate DD radar observations. ${ }^{3}$

The system what we have implemented (Fig. 2) is similar to that of Dresel et al. but is modified to allow us to reproduce the geometries that occur in asteroid radar observations. The source (LD) is a $\lambda=670 \mathrm{~nm}$ Toshiba TOLD 9215 10-mW laser diode. At the power level that we use it has a coherence length measured to be slightly less than $0.2 \mathrm{~mm}$; this sets the minimum range resolution to be $\sim 0.1 \mathrm{~mm}$. The $f$-number of the imaging optics is set large enough that the entire object is brought to focus on a CCD. Polarizer P1 permits sampling of either the copolarized or the crosspolarized component of the field scattered by the object, and polarizer P2 allows the relative amplitude of the reference beam to be adjusted. The target is mounted upon a thin post, which corresponds to its spin axis, and the post is connected to the shaft of a stepper motor that permits user control of the target's subradar longitude, $\psi$. This component is mounted upon a tiltable platform, which allows the subradar latitude $\delta$ to be set as desired.

The position of the reference mirror $(\mathrm{M})$ is controlled by two translation stages. A coarse positioning stage (S1) is used to select the delay resolution cell or range bin. It is driven by a stepper motor with $0.5^{\circ}$ angular resolution turning a 40-pitch screw. This results in a coarse positioning resolution of $882 \mathrm{~nm}$ per step $(\approx 1 / 100$ of the coherence length). A finepositioning stage $(\mathrm{S} 2)$ is used to sample the fringe pattern at the particular range bin of interest. It is driven by a stepper motor with $0.025^{\circ}$ angular resolution turning a 100-pitch screw and is fixed on top of the coarse-positioning stage. The fine positioner provides a resolution of $17.6 \mathrm{~nm}$ per step, which gives approximately 20 samples per fringe period.
At each range bin, the fine positioner is stepped through a complete fringe, with a CCD camera and a computer (PC) frame grabber recording the intensity of each pixel at each step. In this way the amplitude and the phase of the interference fringe pattern for each pixel on the target (e.g., each point $x, y$ in Fig. 1) can be calculated. By noting the mirror position for which the maximum fringe amplitude occurs we obtain $z(x, y)$. Blocking the beam from the reference mirror permits recording of $|b(x, y)|^{2}$, and averaging this for several closely spaced values of $\psi$ gives $\left\langle|b(x, y)|^{2}\right\rangle$; the fringe measurements give the phase of $b(x, y)$. In this manner we obtain all the quantities that we need to calculate the integrals in Eqs. (3) and (6); we are free to choose the period $P$ to simulate any desired set of circumstances. The stepping of the motors and the grabbing of frames are all under software control, so the entire process is fully automatic.

The principal radar telescopes are the Arecibo and Goldstone systems. ${ }^{6}$ Arecibo operates at a primary wavelength $\lambda_{r}=12.6 \mathrm{~cm}$ and currently has a minimum delay resolution of $1 / 8 \mu \mathrm{s}$ or $18.8 \mathrm{~m}=149 \lambda_{r}$ in range. For the optical wavelength $\lambda=670 \mathrm{~nm}, 149 \lambda=0.1 \mathrm{~mm}$, so, when it is operated with this range resolution, the laser system serves as a $670 \mathrm{~nm} / 12.6 \mathrm{~cm} \times 1 / 188,000$ scale model of the Arecibo radar. Accordingly, a 1-cm rock would correspond to a $1.88-\mathrm{km}$ asteroid, which is, for example, very nearly the maximum dimension of the ECA 4726 Castalia. $^{7}$ Goldstone operates at a primary wavelength of $\lambda_{r}=3.54 \mathrm{~cm}$ and a minimum delay resolution of $1 / 8 \mu \mathrm{s}$, or $18.8 \mathrm{~m}=531 \lambda_{r}$ in range. When operated with a range resolution of $0.36 \mathrm{~mm}$, the laser-radar system can be considered a $1 / 52,800$ scale model of Goldstone. A 1-cm rock then corresponds to a 528-m asteroid, which is close to the size of the ECA $1991 \mathrm{JX}$, recently imaged at Goldstone at $1 / 8 \mu \mathrm{s}$.

As an example of the use of this system, Fig. 3A shows optical-radar DD and POS images of a 1.1-cm bolt. The bolt was painted with white correction fluid to produce a rough and reflective surface. The latitude is $\delta=0$; two longitudes are shown, $0^{\circ}$ and $45^{\circ}$. The DD images have resolutions of $0.1 \mathrm{~mm}$ in both dimensions and were calculated from Eq. (6) with 20 realizations of $|b(x, y)|^{2}$, each at a slightly different value of $\psi$, averaged to estimate $\left\langle|b(x, y)|^{2}\right\rangle$.

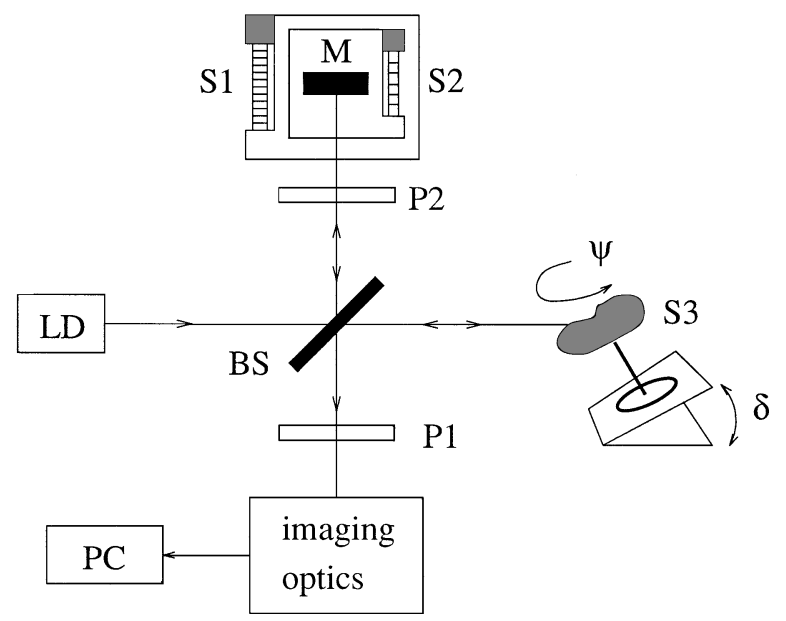

Fig. 2. Laser-radar system. BS, beam splitter. 

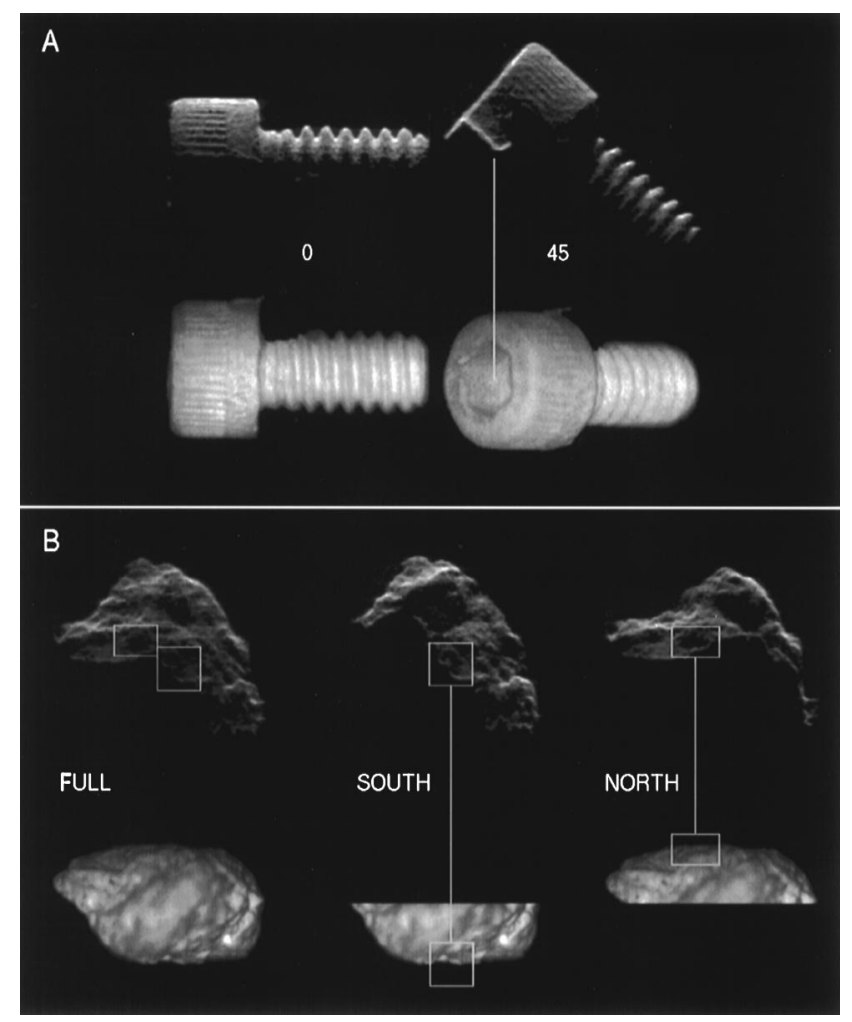

Fig. 3. A, DD (top row) and POS (bottom row) images of a 1.1-cm bolt. Delay increases from top to bottom; Doppler increases from right to left. B, DD and POS images of a 1-cm rock.

These images demonstrate some of the geometric differences between the POS and DD imaging geometries. One of the most interesting is how concavities in the object can produce gaps in the DD image. For example, in the $\psi=45^{\circ}$ DD image the bolt head and the threaded shaft appear disconnected, whereas they are joined in the POS image. This is due to the discontinuity in range in going from the bolt head to the shaft and to the fact that the bolt head shadows the part of the bolt that joins these two pieces. The hex-head concavity in the bolt (pointed to by the vertical line in $45^{\circ}$ images) appears quite different in the POS and DD images because of the different imaging geometry.

An example of optical DD imaging of a natural object is given in Fig. 3B. Here the object is an $\sim 1$-cm basalt rock, which is very rough and pitted. DD images at 0.1-mm resolution are shown for the entire object and for the top (north) and bottom (south) halves separately. Again we see how surface concavities produce shadows in the DD image. This image sequence also illustrates the north-south ambiguity. In the full image, two DD features are boxed. Their proximity in the image might be taken to imply that they are adjacent features on the surface of the object. However, examination of the DD images of the north and south halves separately shows that, in fact, the corresponding surface features are on opposite ends of the object. This illustrates the difficulty that one would encounter in trying to directly interpret DD images by eye.

We have demonstrated how one can use a laboratory optical-radar system to obtain realistic scalemodel planetary radar data sets analogous to the highest-resolution radar data available for asteroids. With this system data can be conveniently obtained for arbitrary centimeter-sized models, allowing us to investigate the relationship between DD images and three-dimensional shape and to produce realistic test cases for planetary radar data-processing techniques.

\section{References}

1. C. R. Chapman and D. Morrison, Nature (London) 367, 33 (1994).

2. T. D. Jones, D. B. Eppler, D. R. Davis, A. L. Friedlander, J. McAdams, and S. Krikalev, in Hazards Due to Comets and Asteroids, T. Gehrels, ed. (U. Arizona Press, Tucson, 1994), pp. 683-708.

3. R. S. Hudson, Remote Sensing Rev. 8, 195 (1993).

4. S. J. Ostro, in Asteroids II, R. P. Binzel, T. Gehrels, and M. S. Matthews, eds. (U. Arizona Press, Tucson, Ariz., 1989), pp. 192-212.

5. T. Dresel, G. Hausler, and H. Venzke, Appl. Opt. 31, 919 (1992).

6. S. J. Ostro, Rev. Mod. Phys. 65, 1235 (1993).

7. R. S. Hudson and S. J. Ostro, Science 263, 940 (1994). 